\title{
UNIFORM LIMITS OF CERTAIN $A$-HARMONIC FUNCTIONS WITH APPLICATIONS TO QUASIREGULAR MAPPINGS
}

\author{
Alexandre Eremenko and John L. Lewis
}

\begin{abstract}
Let $u_{1}, u_{2}, \ldots, u_{m}$ be nonconstant uniform limits (on compact subsets) of $A$ harmonic functions in $\{x:|x|<R\} \subset \mathbf{R}^{n}$ where $A$ satisfies certain elliptic structure conditions. The authors show that if there exists $\lambda \geq 0$ such that (i) $\left\{x: u_{i}(x)<-\lambda\right\} \cap\left\{x: u_{j}(x)<-\lambda\right\}=\emptyset$, (ii) $\left|u_{j}^{+}-u_{i}^{+}\right| \leq \lambda$, and (iii) $\left|u_{j}(0)\right| \leq \lambda$, for $1 \leq i, j \leq m$, then $m \leq c$ where $c$ depends only on the structure conditions and $n$. As an application they show that their theorem provides a completely P.D.E. proof of Rickman's generalization of Picard's theorem to quasiregular mappings.
\end{abstract}

\section{Introduction}

Let $x=\left(x_{1}, \ldots, x_{n}\right)$ denote a point in Euclidean $n$ space $\left(\mathbf{R}^{n}\right)$, and put

$$
\begin{gathered}
\langle x, y\rangle=\sum_{i=1}^{n} x_{i} y_{i}, \quad x, y \in \mathbf{R}^{n}, \\
B(x, r)=\{y:|y-x|<r\}, \quad r>0, x \in \mathbf{R}^{n} .
\end{gathered}
$$

Let $\bar{E}, \partial E$, and $|E|$ denote the closure, boundary, and Lebesgue $n$ measure of $E$. If $g$ is a function on $\mathbf{R}^{n}$, put $M\left(r, g, x_{0}\right)=\sup _{B\left(x_{0}, r\right)} g, g^{+}=\max (g, 0)$ and

$$
\nabla g=\left(\frac{\partial g}{\partial x_{1}}, \frac{\partial g}{\partial x_{2}}, \ldots, \frac{\partial g}{\partial x_{n}}\right)
$$

Let $L_{p}(\Omega), 1 \leq p \leq \infty$, be the usual space of Lebesgue measurable functions $g$ on a domain $\Omega$ with norm denoted by $\|g\|_{p}$. Let $W_{1, p}(\Omega)$ be the Sobolev space of functional elements with distributional gradients $\nabla g$ and norm given by $\|g\|_{1, p}=\|\nabla g\|_{p}+\|g\|_{p}$. We say that $g \in W_{1, p}(\Omega)$ locally, provided $g \in W_{1, p}(O)$ whenever $O$ is an open set with $\bar{O} \subseteq \Omega$. We denote the space of functions with compact support in $\Omega$ by $C_{0}^{\infty}(\Omega)$, and set $\dot{W}_{1, p}(\Omega)$, equal to the closure in $W_{1, p}(\Omega)$ of $C_{0}^{\infty}(\Omega)$.

For fixed $p, 1<p<\infty$, suppose that $A=A(x, \eta)$ is a function from $\Omega \times \mathbf{R}^{n} \rightarrow \mathbf{R}^{n}$ with the following properties:

Supported by NSF and the Commonwealth of Kentucky through the Kentucky EPSCoR program.

1980 Mathematics Subject Classification (1985 Revision): Primary 35B60, 30C60. 
(a) For each $\eta \in \mathbf{R}^{n}, x \rightarrow A(x, \eta)$ is Lebesgue measurable,

(b) For almost every $x \in \Omega$ (with respect to Lebesgue $n$ measure) $\eta \rightarrow A(x, \eta)$ is continuous,

(c) $c_{1}(|\xi|+|\eta|)^{(p-2)}|\xi-\eta|^{2} \leq\langle A(x, \eta)-A(x, \xi), \eta-\xi\rangle$, for almost every $x \in \mathbf{R}^{n}$, whenever $\xi \in \mathbf{R}^{n}, \eta \in \mathbf{R}^{n}-\{0\}$,

(d) $|A(x, \eta)| \leq c_{2}|\eta|^{p-1}$ for almost every $x \in \Omega$, whenever $\eta \in \mathbf{R}^{n}$.

$v$ is said to be a weak solution to $\nabla \cdot[A(x, \nabla v(x))]=0$ in $\Omega$, provided $v$ is continuous on $\Omega$, locally in $W_{1, p}(\Omega)$ and

$$
\int_{\Omega}\langle A(x, \nabla v), \nabla \psi\rangle d x=0
$$

when $\psi \in C_{0}^{\infty}(\Omega)$. Note from (d) and Hölder's inequality that (1.1) remains true for $\psi \in \dot{W}_{1, p}(O)$, whenever $O$ is open and $\bar{O} \subseteq \Omega$. We remark that solutions $v$ as above are often called $A$-harmonic in the literature.

Next for fixed $a, b, p_{1}, p_{2}$ with $0<a, b<\infty$, and $1<p_{1}<p_{2}<\infty$ let $F\left(a, b, p_{1}, p_{2}, \Omega\right)$ denote the space of functions $w$ on $\Omega$ which are uniform limits (on compact subsets of $\Omega$ ) of sequences $\left(v_{k}\right)_{1}^{\infty}$ satisfying: Each $v_{k}$ is a weak solution to $\nabla \cdot A^{(k)}\left(x, \nabla v_{k}(x)\right)=0$ in $\Omega$; where $A^{(k)}$ satisfies (a)-(d) with constants $c_{1}(k), c_{2}(k), p(k)$. Moreover, $a \leq c_{1}(k), b \geq c_{2}(k)$ and $p_{1} \leq p(k) \leq p_{2}$ for $k=1,2, \ldots$ In this paper we prove in Section 3 :

Theorem 1. For fixed $a, b, p_{1}, p_{2}$ as above let $u_{1}, u_{2}, \ldots, u_{m}$ be nonconstant functions in $F\left[a, b, p_{1}, p_{2}, B(0, R)\right]$. Suppose for some $\lambda \geq 0$ that whenever $1 \leq i, j \leq m, i \neq j$,

(i) $\left\{x: u_{i}(x)<-\lambda\right\} \cap\left\{x: u_{j}(x)<-\lambda\right\}=\emptyset$,

(ii) $\left|u_{i}^{+}-u_{j}^{+}\right| \leq \lambda$,

(iii) $\left|u_{j}(0)\right| \leq \lambda$.

Then there exists $c_{3}=c_{3}\left(n, a, b, p_{1}, p_{2}\right)>0$ such that either

$$
m \leq c_{3}
$$

or

$$
M\left(\frac{R}{100}, u_{j}, 0\right) \leq c_{3} \lambda, \quad 1 \leq j \leq m .
$$

Observe that $c_{3}$ in Theorem 1 is independent of $\lambda$ and $R$. Using this observation, we easily obtain the following Corollary (see Section 3 ).

Corollary 1. Let $a, b, p_{1}, p_{2}$, be as in Theorem 1 and suppose $u_{1}, u_{2}, \ldots, u_{m}$ are nonconstant functions in $F\left(a, b, p_{1}, p_{2}, \mathbf{R}^{n}\right)$. Suppose for some $\lambda \geq 0$ that (i)-(iii) of Theorem 1 are valid. Then (1.2) holds. 
We note that Corollary 1 follows directly from Theorem 1, once we show $u_{1}, \ldots, u_{m}$ are unbounded above in $\mathbf{R}^{n}$. Theorem 1 and Corollary 1 , with $p_{1}=$ $p_{2}=n$, have applications to quasiregular mappings. Recall that a function $f$ from a domain $\Omega$ into $\mathbf{R}^{n}$ is said to be $K>1$ quasiregular provided each of its coordinate functions are locally in $W_{1, n}(\Omega)$ and

$$
|D f(x)|=\sup _{|h|=1}|D f(x) h| \leq K J_{f}(x)
$$

for almost every $x \in \Omega$. Here $D f(x)$ is the Jacobian matrix of $f$ while $J_{f}$ is the determinant of $D f$ (the Jacobian of $f$ ). Moreover, if $f \neq d$ in $\Omega$ then $u=\log |f-d|$ is locally a weak solution to (see [1])

$$
\nabla \cdot\left[\langle A(x) \nabla u(x), \nabla u(x)\rangle^{(n-2) / 2} A(x) \nabla u(x)\right]=0
$$

in $\Omega$, where

$$
A(x)=J_{f}(x)^{2 / n}\left[D^{t} f(x) D f(x)\right]^{-1},
$$

when $D f(x)^{-1}$ exists, and $A(x)=$ identity matrix, otherwise. In (1.5) $D^{t} f$ denotes the transpose matrix of $D f$. Now if

$$
A(x, \eta)=\langle A(x) \eta, \eta\rangle^{(n-2) / 2} A(x) \eta,
$$

$(x, \eta) \in \Omega \times \mathbf{R}^{n}$, then clearly (a) and (b) are valid. (d) for $p=n$ is a consequence of (1.4) with $c_{2}=c_{2}(n, K)$. (c) follows from the case $p=n$ of the inequality

$$
(|y|+|w|)^{p-2}|y-w|^{2} \leq c\left\langle|y|^{p-2} y-|w|^{p-2} w, y-w\right\rangle,
$$

where $1<p<\infty, c=c(n, p)$ and $y \in \mathbf{R}^{n}, w \in \mathbf{R}^{n}-\{0\}$. To get (d) from (1.8) put

$$
\theta(x)=J_{f}(x)^{1 / n} D f(x)^{-1},
$$

when $D f(x)^{-1}$ exists, and $\theta(x)=$ identity matrix, otherwise. Then from (1.7) and (1.8) with $y=\theta(x) \eta, w=\theta(x) \xi$ we deduce (c) for $c_{1}=c_{1}\left(n, K^{\prime}\right)>0$ small enough. Hence $u \in F(a, b, n, n, \Omega)$ for some $a=a(n, K), b=b(n, K)$.

Next we use the above facts about quasiregular mappings and Corollary 1 to prove Rickman's theorem [12]: A nonconstant entire quasiregular mapping omits at most a finite number of values. To this end suppose $f$ is a nonconstant entire quasiregular mapping which omits distinct values $a_{1}, \ldots, a_{m}$. Let $u_{i}=\log \left|f-a_{i}\right|$, $1 \leq i \leq m$, and choose $\delta, 0<\delta \leq 1$, so small that

$$
0<\delta \leq \min \left\{\left|f(0)-a_{i}\right|,\left|a_{i}-a_{j}\right|\right\} \leq \max \left\{\left|f(0)-a_{i}\right|,\left|a_{i}-a_{j}\right|\right\} \leq \delta^{-1},
$$


when $i \neq j, 1 \leq i, j \leq m$. Then from the triangle inequality and simple properties of logarithms it is easily seen that (i)-(iii) of Theorem 1 hold with $\lambda=2 \log 2+\log (1 / \delta)$. Thus we can apply Corollary 1 to get $m \leq c(n, K)$, which is Rickman's theorem for nonconstant entire quasiregular mappings.

We note that Rickman in [15] also proved a version of Schottky's theorem for quasiregular mappings. A somewhat weaker but similar theorem can be deduced from Theorem 1 by essentially the same argument as above. We omit the details. Now suppose that $u_{1}, \ldots, u_{m}$ are as in Theorem 1 and $\lambda=0$ in (i)-(iii). Then $m \leq c_{3}$, since otherwise it would follow from (1.3), Harnack's inequality for positive functions in $F\left[a, b, p_{1}, p_{2}, B(0, R)\right]$ (see Lemma 3 ) and a connectivity argument that $u_{1} \equiv \cdots \equiv u_{m} \equiv 0$. Suppose $m \geq 2$ and put

$$
\begin{aligned}
O_{1} & =\left\{x: u_{1}^{+}(x)>0\right\}, \\
O_{i+1} & =\left\{x: u_{i}(x)<0\right\}, \quad 1 \leq i \leq m .
\end{aligned}
$$

Using Harnack's inequality for $F\left(a, b, p_{1}, p_{2}, B(0, R)\right)$ again, it is easily seen that

$$
B(0, R) \cap \partial O_{i}=B(0, R) \cap \partial O_{j}, \quad 1 \leq i, j \leq m+1 .
$$

Such an equality between open sets is topologically possible, but it requires some work to construct examples since $m \geq 2$. In Section 3 we point out that $m \geq 2$ is in fact possible in Theorem 1 when $\lambda=0$. However, we do not know whether there exist $u_{1}, \ldots, u_{m}(m>1)$ which are $A$-harmonic for some $A=A(x, \eta)$ and satisfy the conditions of Theorem 1 with $\lambda=0$. A similar problem is to characterize those $A=A(x, \eta)$ satisfying: If $u, v$ are $A$ harmonic in $B(0, R)$ with $u(0)=0, u^{+}=v^{+}$, and $\{u<0\} \cap\{v<0\}=\emptyset$, then $u \equiv v \equiv 0$. This problem is related to (but not the same as) the problem of determining those $A$ which have the unique continuation property at 0 : If $u, v$ are $A$-harmonic in $B(0, R)$ and $u=v$ on an open set $O \subseteq B(0, R)$, then $u \equiv v$ in $B(0, R)$ (see [5], [7], [18] for references and recent results in unique continuation). Another interesting question which arises from this paper is to find minimal structural assumptions on $A$ which guarantee that the conclusion of Theorem 1 or Corollary 1 is valid when $u_{1}, \ldots, u_{m}$ are $A$-harmonic and satisfy (i)-(iii).

As for the proof of Theorem 1, first observe that if $u_{1}, \ldots, u_{m}$ satisfy (i)(iii) with $\lambda=0$, then these functions satisfy (i)-(iii) for each positive $\lambda$. Hence Theorem 1 for $\lambda=0$ follows from Theorem 1 for $\lambda>0$ and so we assume throughout the proof of Theorem 1 that $\lambda>0$. Second observe from the definition of $F\left(a, b, p_{1}, p_{2}, B(0, R)\right)$ and (iii) that there exists an $A=A(x, \eta)$ harmonic $u$ in $B(0, R)$ with

$$
u(0)=0
$$

and

$$
\left|u(x)-u_{1}(x)\right| \leq 2 \lambda, \quad x \in B\left(0, \frac{9}{10} R\right)
$$


Let $p, p_{1} \leq p \leq p_{2}$ be the exponent in (c), (d) corresponding to $u$ and let $\mu$ be the unique positive Borel measure associated with $u^{+}$as follows:

$$
\int_{B(0, R)}\left\langle A\left(x, \nabla u^{+}\right), \nabla \phi\right\rangle d x=-\int_{B(0, R)} \phi d \mu,
$$

whenever $\phi \in C_{0}^{\infty}(B(0, R))$. The existence of $\mu$ can be deduced as in [6].

For completeness we sketch a proof of (1.11) in Section 2. Let

$$
n\left(t, x_{1}\right)=t^{p-n} \mu\left(B\left(x_{1}, t\right)\right), \quad t>0 .
$$

Next in Section 2 we prove

Lemma 1. Let $x_{1} \in B(0, R / 4) \cap\{x: u(x)=0\}$, and $0<\rho \leq R / 100$. Then there exists $c_{4}=c_{4}\left(n, a, b, p_{1}, p_{2}\right)>0$ such that

$$
\left(c_{4}\right)^{-1} n\left(\rho, x_{1}\right) \leq M\left(2 \rho, u, x_{1}\right)^{p-1} \leq M\left(5 \rho, u, x_{1}\right)^{p-1} \leq c_{4} n\left(10 \rho, x_{1}\right) .
$$

In Section 3 we establish

Lemma 2. There exists $r<10^{-4} R, x_{2} \in B(0, R / 4) \cap\{x: u(x)=0\}$, and $c_{5}=c_{5}\left(n, a, b, p_{1}, p_{2}\right)>0$, such that

$$
\max \left\{n(R / 8,0), n\left(10 r, x_{2}\right)\right\} \leq c_{5} n\left(r, x_{2}\right) .
$$

We observe from (ii), (1.10), Lemma 1 with $x_{2}=x_{1}, \rho=r$ and Lemma 2 that

$$
\begin{aligned}
M\left(5 r, u_{i}^{+}, x_{2}\right)-3 \lambda & \leq M\left(5 r, u, x_{2}\right) \leq\left[c_{4} n\left(10 r, x_{2}\right)\right]^{1 /(p-1)} \\
& \leq\left[c_{4} c_{5} n\left(r, x_{2}\right)\right]^{1 /(p-1)} \leq\left(c_{4}^{2} c_{5}\right)^{1 /(p-1)} M\left(2 r, u, x_{2}\right) \\
& =c_{6} M\left(2 r, u, x_{2}\right) \leq c_{6}\left[M\left(2 r, u_{i}{ }^{+}, x_{2}\right)+3 \lambda\right],
\end{aligned}
$$

when $1 \leq i \leq m$. Similarly, using (ii), (1.9)-(1.10), Lemma 1 with $x_{1}=0$, $\rho=R / 100$ and Lemma 2 , we get

$$
\begin{aligned}
M\left(\frac{R}{100}, u_{i}{ }^{+}, 0\right)-3 \lambda & \leq M\left(\frac{R}{100}, u, 0\right) \leq\left[c_{4} n(R / 8,0)\right]^{1 /(p-1)} \\
& \leq\left[c_{4} c_{5} n\left(r, x_{2}\right)\right]^{1 /(p-1)} \leq c_{6} M\left(2 r, u, x_{2}\right) \\
& \leq c_{6}\left[M\left(2 r, u_{i}^{+}, x_{2}\right)+3 \lambda\right] .
\end{aligned}
$$

Next in Section 3 we show that (1.12), (1.13) and essentially Harnack's inequality for $F\left[a, b, p_{1}, p_{2}, B(0, R)\right]$ (see Lemma 3 ) imply the existence of $c_{7}=$ $c_{7}\left(n, a, b, p_{1}, p_{2}\right)>0$, such that

$$
\begin{aligned}
\left(c_{7}\right)^{-2}\left[M\left(\frac{R}{100}, u_{j}^{+}, 0\right)-2\left(c_{7}\right)^{3} \lambda\right] & \leq\left(c_{7}\right)^{-1}\left[M\left(2 r, u, x_{2}\right)-\left(c_{7}\right)^{2} \lambda\right] \\
& \leq M\left(4 r,-u_{i}, x_{2}\right) \leq c_{7}\left[M\left(3 r,-u_{i}, x_{2}\right)+\lambda\right] \\
& \leq c_{7}^{2}\left[M\left(5 r, u, x_{2}\right)+\lambda\right]
\end{aligned}
$$


holds for $1 \leq i, j \leq m$, except for at most one possible $i$. In Section 3 we also deduce from (1.14) that if (1.3) is false for some $j, 1 \leq j \leq m$, and $c_{3}$ is large enough, then

$$
c_{8}\left|\left\{x \in B\left(x_{2}, 4 r\right):-u_{i}(x)>\frac{1}{2} M\left(3 r,-u_{i}, x_{2}\right)>\lambda\right\}\right| \geq r^{n}
$$

is valid for $1 \leq i \leq m$, with at most one exception. Here $c_{8}=c_{8}\left(n, a, b, p_{1}, p_{2}\right)$ is a small positive constant. Since by assumption $\left\{x: u_{i}(x)<-\lambda\right\}, 1 \leq i \leq m$ are pairwise disjoint, we can then conclude from (1.15) that (1.2) and hence Theorem 1 are true.

As motivation for the proof of Theorem 1 we note that Rickman used a somewhat similar format in [12]. However Lemma 2 is simpler than its corresponding analogue (Lemma 5.1) in [12]. Also much of the proof in [12], [15] uses the "method of moduli of path families". This method uses extremal length and many facts about quasiregular mappings which are not available here. We remark that one of our goals in writing this paper was to make Rickman's generalization of Picard's theorem (which in our opinion is one of the highpoints in the the theory of quasiregular mappings) more accessible to a larger audience, e.g. a person who is an expert in p.d.e.'s and a nonexpert in q.r. mappings. Theorem 1 is also motivated by work of Eremenko and Sodin in [2,3]. They showed that Nevanlinna's second fundamental theorem follows from a theorem they proved for $\delta$-subharmonic functions, and in fact this theorem was used in [2-4] to prove a conjecture of Shiffman and give a simple proof of Drasin's theorem on deficient values. Rephrasing Rickman's Theorem in the language of $A$-harmonic functions led us to conjecture Theorem 1 . In a future paper we hope to obtain a generalization of the defect relation in [13] to " $\delta$ - $A$-subharmonic functions".

Finally, we would like to thank Juha Heinonen for some helpful conversations. Also the authors would like to thank Tero Kilpeläinen and Jan Mály for a preprint of [9] which was helpful in proving Theorem 1.

\section{Preliminary reductions}

In the sequel $c$ denotes a positive constant which may only depend on $n, a$, $b, p_{1}, p_{2}$, not necessarily the same at each occurence. Here we list some facts about $A$-harmonic functions which will be used in the proof of Theorem 1. Let $v \geq 0, w$ be $A$-harmonic in $B(y, t), t>0, y \in \mathbf{R}^{n}$ with constants $p=q, c_{1}, c_{2}$, in (c), (d), satisfying $c_{1} \geq a, c_{2} \leq b$ and $p_{1} \leq q \leq p_{2}$. Then we shall often use Harnack's inequality for $A$-harmonic functions:

$$
M(\tau, v, y) \leq c\left(\frac{t}{t-\tau}\right)^{\beta} \min _{B(y, \tau)} v,
$$

where $0<\tau<t$ and $\beta=\beta\left(n, a, b, p_{1}, p_{2}\right) \geq 1$. We refer the reader to [17, Theorem 5] for a proof of (2.1) when $\tau=\frac{1}{2} t$. (2.1) can be deduced from this case 
by iteration. Using (2.1), it is easily shown that there exists $\alpha=\alpha\left(n, a, b, p_{1}, p_{2}\right)$, $0<\alpha<1$ such that

$$
\begin{aligned}
\underset{B\left(y, t_{1}\right)}{\operatorname{osc} w} & =\sup _{x, z \in B\left(y, t_{1}\right)}|w(x)-w(z)| \\
& =M\left(t_{1}, w, y\right)+M\left(t_{1},-w, y\right) \leq c\left(t_{1} / t_{2}\right)^{\alpha} \underset{B\left(y, t_{2}\right)}{\operatorname{osc}} w \\
& =c\left(t_{1} / t_{2}\right)^{\alpha}\left[M\left(t_{2}, w, y\right)+M\left(t_{2},-w, y\right)\right],
\end{aligned}
$$

when $0<t_{1}<t_{2}<t$. To obtain (2.2) from (2.1), put $s=t, 9 s / 10=\tau$ and $v=M(s, w, y) w, v=M(s,-w, y)+w$ respectively in (2.1). Adding the resulting inequalities, we find for some $\delta=\delta\left(n, a, b, p_{1}, p_{2}\right), 0<\delta<1$, that

$$
M\left(\frac{9}{10} s, w, y\right)+M\left(\frac{9}{10} s,-w, y\right) \leq \delta[M(s, w, y)+M(s,-w, y)]
$$

which can be iterated to get (2.2). Similarly, if $v=M(9 t / 10, w, y)-w$ then from (2.1) we have with $t$ replaced by $9 t / 10$ and $\tau$ by $4 t / 5$,

$$
M\left(\frac{9}{10} t, w, y\right)+M\left(\frac{4}{5} t, w, y\right) \leq c\left[M\left(\frac{9}{10} t, w, y\right)-w(y)\right]
$$

Thus

$$
M\left(\frac{4}{5} t,-w, y\right) \leq(c-1) M\left(\frac{9}{10} t, w, y\right)-c w(y) .
$$

We observe that (2.3) is also valid if $w$ is replaced by $-w$, since if $w$ is $A(x, \eta)$ harmonic, then $-w$ is $A(x,-\eta)$-harmonic. Finally observe from (a)-(c) that $w+s$ is $A(x, \eta)$ harmonic for each real number $s$. From these observations and (2.1)(2.3) we easily deduce the following lemma.

Lemma 3. Let $v \geq 0, w \in F\left[a, b, p_{1}, p_{2}, B(y, t)\right]$. Then $-w, w+s$ are in $F\left[a, b, p_{1}, p_{2}, B(y, t)\right]$. Also $v$ satisfies (2.1) while $w$ satisfies $(2.2)-(2.3)$.

To prove Lemma 3 it suffices to note that our previous observations and (2.1)(2.3) remain valid for uniform limits.

Next we sketch the proof of existence for $\mu$ as in (1.11). To do this we use a clever idea of Heinonen and Kilpeläinen (see [6, Theorem 3.17]). Fix $R_{1}<R$ and put

$$
K=\left\{\zeta: 0 \leq \zeta \leq u^{+}, \zeta-u^{+} \in \dot{W}_{1, p}\left(B\left(0, R_{1}\right)\right)\right\} .
$$

Using (a)-(d) and the theory of monotone coercive operators (see Theorem 1.7 and Section 4 in Chapter 3 of [8]) we deduce the existence of $v \in K$ with

$$
\int_{B\left(0, R_{1}\right)}\langle A(x, \nabla v), \nabla \phi\rangle d x \leq 0
$$


whenever $0 \leq \phi \in \dot{W}_{1, p}\left(B\left(0, R_{1}\right)\right)$. $v$ is said to be a solution to the obstacle problem for $A$ in $B\left(0, R_{1}\right)$, with obstacle and boundary function equal to $u^{+}$. Using Hölder continuity of $u^{+}$(see (2.2)) and Moser iteration, we conclude in a standard way (see [11, Theorem 3.7]) that $v$ is Hölder continuous in $B\left(0, R_{1}\right)$ and $A$-harmonic in

$$
O=\left\{x \in B\left(0, R_{1}\right): v(x)<u^{+}(x)\right\} .
$$

Since $v, u^{+}$are $A$-harmonic in $O$ and $v-u^{+} \in \dot{W}_{1, p}(O)$ we deduce from the maximum principle for $A$-harmonic functions, implied by (c) in Section 1 (see [6]), that $v \equiv u^{+}$. Since $R_{1}, 0<R_{1}<R$ is arbitrary we conclude that (2.4) holds with $v=u^{+}$, whenever $0 \leq \phi \in \dot{W}_{1, p}(G)$ and $G$ is open with $\bar{G} \subseteq B(0, R)$. From (2.4) and the Riesz representation theorem we now get (1.11).

To begin the proof of Lemma 1 let $0 \leq \sigma \in C_{0}^{\infty}\left[B\left(x_{1}, t\right)\right]$ with $\sigma \equiv 1$ on $B\left(x_{1}, s\right)$ and $|\nabla \sigma| \leq 100(t-s)^{-1}, 0<s<t \leq 10 \rho$. Put $v=u, \psi=u^{+} \sigma^{p}$ in (1.1). From (c) in Section 1 with $\xi=0$ we obtain

$$
\begin{aligned}
\int_{B\left(x_{1}, s\right)}\left|\nabla u^{+}\right|^{p} d x & \leq c \int_{B\left(x_{1}, t\right)}\left(\left|\nabla u^{+}\right| \sigma\right)^{p} d x \\
& \leq c \int_{B\left(x_{1}, t\right)}\left\langle A\left(x, \nabla u^{+}\right), \sigma^{p} \nabla u^{+}\right\rangle d x=J_{1} .
\end{aligned}
$$

From (1.1) and (d) we observe that

$$
0=\int_{B\left(x_{1}, t\right)}\left\langle A(x, \nabla u), \nabla\left(u^{+} \sigma^{p}\right)\right\rangle d x=\int_{B\left(x_{1}, t\right)}\left\langle A\left(x, \nabla u^{+}\right), \nabla\left(u^{+} \sigma^{p}\right)\right\rangle d x .
$$

From this observation, (d), Hölder's inequality and (2.5) we deduce

$$
\begin{aligned}
J_{1} & =-c \int_{B\left(x_{1}, t\right)}\left\langle A\left(x, \nabla u^{+}\right), u^{+} \nabla \sigma^{p}\right\rangle d x \\
& \leq c \int_{B\left(x_{1}, t\right)}\left|\nabla u^{+}\right|^{p-1} \sigma^{p-1} u^{+}|\nabla \sigma| d x \leq c J_{1}^{1-1 / p} J_{2}{ }^{1 / p}
\end{aligned}
$$

where

$$
J_{2}=\int_{B\left(x_{1}, t\right)}\left(u^{+}|\nabla \sigma|\right)^{p} d x \leq c(t-s)^{-p} t^{n} M\left(t, u, x_{1}\right)^{p} .
$$

From (2.6) we see that $J_{1} \leq c J_{2}$. Putting this inequality, (2.5) and (2.7) together, we get

$$
\int_{B\left(x_{1}, s\right)}\left|\nabla u^{+}\right|^{p} d x \leq c(t-s)^{-p} t^{n} M\left(t, u, x_{1}\right)^{p}
$$


Next, let $s=\rho, t=3 \rho / 2$ and put $\phi \equiv \sigma$ in (1.11). Again from (c), (d), and Hölder's inequality we find

$$
\begin{aligned}
\mu\left(B\left(x_{1}, \rho\right)\right) & \leq \int \sigma d \mu=-\int\left\langle A\left(x, \nabla u^{+}\right), \nabla \sigma\right\rangle d x \\
& \leq c \int\left|\nabla u^{+}\right|^{p-1}|\nabla \sigma| d x \leq c\left[\int_{\left(B\left(x_{1}, 3 \rho / 2\right)\right.}\left|\nabla u^{+}\right|^{p} d x\right]^{1-1 / p} \rho^{(n / p-1)} \\
& \leq c \rho^{n-p} M\left(2 \rho, u, x_{1}\right)^{p-1},
\end{aligned}
$$

where we have used (2.8) in the last inequality, with $t=2 \rho, s=3 \rho / 2$. Thus the left hand inequality in Lemma 1 is valid.

To prove the right hand inequality in Lemma 1, we note from $(2.2),(2.3)$ of Lemma 3 with $w=u, y=x_{1}$, that

$$
M\left(t_{1}, u, x_{1}\right) \leq c\left(t_{1} / t_{2}\right)^{\alpha} M\left(t_{2}, u, x_{1}\right),
$$

whenever $0<t_{1} \leq t_{2} \leq 10 \rho$. We now consider two cases. If $p \geq 2$, let $h$ be the $A$ harmonic function in $B\left(x_{1}, 10 \rho\right)$ with $h-u^{+} \in \dot{W}_{1, p}\left(B\left(x_{1}, 10 \rho\right)\right)$. Again, existence of $h$ follows from the theory of monotone coercive operators. Also $0 \leq u^{+} \leq h$ as follows from the maximum principle for $A$-harmonic functions (see [6]). Using these facts and (2.1) of Lemma 3 with $v=h, t=10 \rho, y=x_{1}$ we find that

$$
M\left(5 \rho, u, x_{1}\right) \leq M\left(5 \rho, h, x_{1}\right) \leq \operatorname{ch}\left(x_{1}\right) \leq c^{2} h(x),
$$

whenever $x \in B\left(x_{1}, 5 \rho\right)$. From (2.10), (2.9) with $t_{2}=5 \rho$ we deduce the existence of $d_{1}=d_{1}\left(n, a, b, p_{1}, p_{2}\right)>0$, such that

$$
M\left(d_{1} \rho, u, x_{1}\right) \leq \frac{1}{2} \min _{\bar{B}\left(x_{1}, 5 \rho\right)} h,
$$

which implies in view of (2.10) for $x \in B\left(x_{1}, d_{1} \rho\right)$ that

$$
d_{2} h\left(x_{1}\right) \leq\left(h-u^{+}\right)(x) \leq d_{3} h\left(x_{1}\right)
$$

where $d_{2}, d_{3}$ have the same dependence as $d_{1}$. Put $\phi=\min \left[h-u^{+}, d_{3} h\left(x_{1}\right)\right]$ in (1.11) and let $L$ be the set of points where $\nabla \phi$ exists and is nonzero. Then from (2.11), Poincaré's inequality, the fact that $h$ is $A$-harmonic, and (c) in Section 1 we find

$$
\begin{aligned}
\left(d_{2} h\left(x_{1}\right)\right)^{p}\left(d_{1} \rho\right)^{n} & \leq c \int \phi^{p} d x \leq c \rho^{p} \int|\nabla \phi|^{p} d x \\
& \leq c \rho^{p} \int_{L}\left(|\nabla h|+\left|\nabla u^{+}\right|\right)^{(p-2)}\left|\nabla h-\nabla u^{+}\right|^{2} d x \\
& \leq c \rho^{p} \int_{L}\left\langle A(x, \nabla h)-A\left(x, \nabla u^{+}\right), \nabla\left(h-u^{+}\right)\right\rangle d x \\
& =-c \rho^{p} \int\left\langle A\left(x, \nabla u^{+}\right), \nabla \phi\right\rangle d x=c \rho^{p} \int \phi d \mu \\
& \leq d_{3} h\left(x_{1}\right) \rho^{p} \mu\left(B\left(x_{1}, 10 \rho\right)\right) .
\end{aligned}
$$


From (2.10) and the above inequality we conclude the righthand inequality in Lemma 1 for $p \geq 2$.

To prove this inequality for $1<p<2$, let $H=H(\cdot, s)$ be the $A$-harmonic function in $B\left(x_{1}, s\right), 5 \rho \leq s<10 \rho$ with $H-u^{+} \in \dot{W}_{1, p}\left(B\left(x_{1}, s\right)\right)$. If $0<s^{\prime}<s$, then from (2.1) we find as in (2.10) that

$$
M\left(s^{\prime}, u, x_{1}\right) \leq M\left(s^{\prime}, H, x_{1}\right) \leq c\left(\frac{s}{s-s^{\prime}}\right)^{\beta} H(x),
$$

whenever $x \in B\left(x_{1}, s^{\prime}\right)$. It follows from (2.13) and (2.9), as in (2.11), that if $s^{\prime \prime}$, $0<s^{\prime \prime}<s^{\prime}$ is chosen so that

$$
c\left(\frac{s^{\prime \prime}}{s^{\prime}}\right)^{\alpha}\left(\frac{s}{s-s^{\prime}}\right)^{\beta}=1
$$

and $c$ is large enough, then

$$
d_{4} H\left(x_{1}\right) \leq\left(H-u^{+}\right)(x) \leq d_{5} H\left(x_{1}\right)
$$

for $x \in B\left(x_{1}, s^{\prime \prime}\right)$, where $d_{4}, d_{5}$ have the same dependence as $d_{1}$ above. Let $\phi=\min \left[H-u^{+}, d_{5} H\left(x_{1}\right)\right]$, and define $L$ relative to $\phi$ as in (2.12). Using (2.15), Poincaré's and Hölder's inequalities, we deduce as in (2.12)

$$
\left(d_{4} H\left(x_{1}\right)\right)^{p}\left(s^{\prime \prime}\right)^{n} \leq c s^{p} \int|\nabla \phi|^{p} d x \leq c s^{p}\left(I_{1}\right)^{p / 2}\left(I_{2}\right)^{1-p / 2} .
$$

Here

$$
I_{1}=\int_{L}\left(\left|\nabla u^{+}\right|+|\nabla H|\right)^{(p-2)}\left|\nabla u^{+}-\nabla H\right|^{2} d x
$$

and

$$
I_{2}=\int_{L}\left(|\nabla H|+\left|\nabla u^{+}\right|\right)^{p} d x
$$

From (c) we have

$$
\begin{aligned}
I_{1} & \leq c \int_{L}\left\langle A(x, \nabla H)-A\left(x, \nabla u^{+}\right), \nabla H-\nabla u^{+}\right\rangle d x \\
& =c \int \phi d \mu \leq d_{5} H\left(x_{1}\right) \mu\left(B\left(x_{1}, 10 \rho\right)\right) .
\end{aligned}
$$

Next, we note from (1.1) with $v=H, \psi=H-u^{+}$, and (c), (d) that

$$
\begin{aligned}
\int_{B\left(x_{1}, s\right)}|\nabla H|^{p} d x & \leq c \int_{B\left(x_{1}, s\right)}\langle A(x, \nabla H), \nabla H\rangle d x \\
& =c \int_{B\left(x_{1}, s\right)}\left\langle A(x, \nabla H), \nabla u^{+}\right\rangle d x \\
& \leq c\left(\int_{B\left(x_{1}, s\right)}\left|\nabla u^{+}\right|^{p} d x\right)^{1 / p}\left(\int_{B\left(x_{1}, s\right)}|\nabla H|^{p} d x\right)^{1-1 / p} .
\end{aligned}
$$


Hence

$$
\int_{B\left(x_{1}, s\right)}|\nabla H|^{p} d x \leq c \int_{B\left(x_{1}, s\right)}\left|\nabla u^{+}\right|^{p} d x
$$

From this inequality and (2.8) we see for $t>s$ that

$$
I_{2} \leq c(t-s)^{-p} t^{n} M\left(t, u, x_{1}\right)^{p} .
$$

Putting the above estimates for $I_{1}, I_{2}$ in (2.16) and using (2.13), we obtain

$$
\begin{aligned}
& M\left(s^{\prime}, u, x_{1}\right)^{p / 2}\left(s^{\prime \prime}\right)^{n} \\
& \quad \leq c\left(\frac{s}{s-s^{\prime}}\right)^{p \beta / 2} t^{a} s^{p} \mu\left(B\left(x_{1}, 10 \rho\right)\right)^{p / 2}(t-s)^{-\theta} M\left(t, u, x_{1}\right)^{\theta},
\end{aligned}
$$

where $\theta=\left(\frac{1}{2} p\right)(2-p)$, and $a=n\left(1-\frac{1}{2} p\right)$. (2.17) can be rewritten, after some juggling, as

$$
\Psi\left(s^{\prime}\right) \leq k\left(s, s^{\prime}, s^{\prime \prime}, t\right)(\Psi(t))^{2-p}
$$

Here

$$
\Psi(\tau)=\left[n\left(10 \rho, x_{1}\right)\right]^{-p / 2(p-1)} M\left(\tau, u, x_{1}\right)^{p / 2}
$$

and

$$
k\left(s, s^{\prime}, s^{\prime \prime}, t\right)=\left(s^{\prime \prime}\right)^{-n}\left(\frac{s}{s-s^{\prime}}\right)^{p \beta / 2} t^{a} s^{p}(t-s)^{-\theta} \rho^{(n-p) p / 2} .
$$

Since $2-p<1$, we can now iterate (2.18) to get the righthand inequality in Lemma 1 when $1<p<2$. Indeed, let

$$
s_{j}=10 \rho\left(1-2^{-j}\right), \quad j=1,2, \ldots,
$$

and put $t=s_{j+1}, s^{\prime}=s_{j}, s=\frac{1}{2}\left(s^{\prime}+t\right)$. Then from (2.14) we deduce that

$$
k\left(s, s^{\prime}, s^{\prime \prime}, t\right) \leq c 2^{j \gamma},
$$

for some $\gamma=\gamma\left(n, a, b, p_{1}, p_{2}\right) \geq 1$. Using this inequality in (2.18) and iterating we get

$$
\Psi\left(s_{1}\right) \leq c 2^{\gamma} \Psi\left(s_{2}\right)^{2-p} \leq \cdots \leq(c 2)^{\left[\gamma+\cdots+j \gamma(2-p)^{j}\right]} \Psi\left(s_{j+1}\right)^{(2-p)^{j}} .
$$

Letting $j \rightarrow \infty$ in (2.19) we conclude first that $\Psi\left(s_{1}\right) \leq c$ and second from the definition of $\Psi$ that the righthand inequality in Lemma 1 is true for $1<p<2$. The proof of Lemma 1 is now complete. 


\section{Proof of Theorem 1}

Next we prove Lemma 2. To this end let $\delta(x)=\frac{1}{4} R-|x|$ when $x \in B\left(0, \frac{1}{4} R\right)$. Let $E=\{x: u(x)=0\} \cap B\left(0, \frac{1}{4} R\right)$ and put

$$
\gamma=\sup \left\{n\left(10^{-4} \delta(x), x\right): x \in E\right\} .
$$

Choose $x_{2} \in E$ such that

$$
n\left(10^{-4} \delta\left(x_{2}\right), x_{2}\right) \geq \frac{1}{2} \gamma
$$

and put $r=10^{-4} \delta\left(x_{2}\right)$. If

$$
y \in E_{1}=E \cap \bar{B}\left(x_{2}, 10 r\right)
$$

then clearly

$$
\frac{1}{2} \delta\left(x_{2}\right) \leq \delta(y) \leq 2 \delta\left(x_{2}\right)
$$

From compactness of $E_{1}$ and a well known covering lemma we see there exist $\left\{y_{i}\right\}_{1}^{l} \in E_{1}$ with

$$
B\left(y_{i}, 10^{-2} r\right) \cap B\left(y_{j}, 10^{-2} r\right)=\emptyset, \quad i \neq j,
$$

and $E_{1} \subseteq \cup_{i=1}^{l} B\left(y_{i}, \frac{1}{4} r\right)$. Now, $l \leq c$, for some absolute constant $c$, as follows from the above equality and a volume argument. Using this fact, (3.1) and (3.2) we deduce that

$$
n\left(10 r, x_{2}\right) \leq c r^{p-n} \mu\left(E_{1}\right) \leq c \sum_{i=1}^{l} n\left(10^{-4} \delta\left(y_{i}\right), y_{i}\right) \leq c \gamma l \leq c_{5} n\left(r, x_{2}\right) .
$$

Similarly, $\bar{B}(0, R / 8) \cap E$ can be covered by at most $c$ balls of radius $10^{-6} R$ whose centers are in $E$. Using (3.1) again on each ball and summing we get

$$
n(R / 8,0) \leq c R^{p-n} \mu\left(\bar{B}\left(0, \frac{R}{8}\right) \cap E\right) \leq c_{5} n\left(r, x_{2}\right) .
$$

From this inequality and (3.3) we conclude that Lemma 2 is valid.

We now prove Theorem 1. We follow the procedure outlined in Section 1. It follows from Lemmas 1 and 2 as in (1.12), (1.13) that

$$
\max \left\{M(R / 8, u, 0), M\left(10 r, u, x_{2}\right)\right\} \leq c M\left(2 r, u, x_{2}\right) .
$$


From (ii) of Theorem 1 and (1.10) we see that $\left|u_{i}{ }^{+}-u^{+}\right| \leq 3 \lambda$ for $1 \leq i \leq m$. Using this inequality and (3.4) we get

$$
\max \left\{M\left(R / 8, u_{i}{ }^{+}, 0\right), M\left(10 r, u_{i}{ }^{+}, x_{2}\right)\right\} \leq c\left[M\left(2 r, u_{i}{ }^{+}, x_{2}\right)+3 \lambda\right] .
$$

We note that (3.5) is essentially equivalent to (1.12)-(1.13). Next let $\Lambda$ be the set of all $i, 1 \leq i \leq m$ with

$$
-\lambda \leq u_{i}\left(x_{2}\right) \leq 3 \lambda
$$

We observe from $u\left(x_{2}\right)=0$ (i), (ii), and (1.10) that the cardinality of $\Lambda$ is at least $m-1$. From this observation and (2.3) of Lemma 3 with $w=u_{i}, y=x_{2}$, and $50 r=9 t$, we deduce for $i \in \Lambda$

$$
M\left(4 r,-u_{i}, x_{2}\right) \leq c\left[M\left(5 r, u_{i}, x_{2}\right)+4 \lambda\right] .
$$

Similarly, using the above observation and (2.3) of Lemma 3 with $w=-u_{i}$, $y=x_{2}$, and $30 r=9 t$ we get for $i \in \Lambda$,

$$
M\left(2 r, u_{i}, x_{2}\right) \leq c\left[M\left(3 r,-u_{i}, x_{2}\right)+4 \lambda\right] .
$$

We observe that (3.5)-(3.7) imply (1.14) for $i \in \Lambda$. Also if $M\left(R / 100, u_{j}, 0\right)>c_{3} \lambda$ for some $j, 1 \leq j \leq m$, and $c_{3}$ is large enough, then from (3.5)-(3.7) we see that

$$
4 \lambda \leq M\left[* u_{i}, s, x_{2}\right] \leq c M\left[{ }^{\prime} u_{k}, t, x_{2}\right],
$$

whenever $i, k \in \Lambda, 3 r \leq s, t \leq 4 r$ and $*,{ }^{\prime} \in\{+,-\}$. For fixed $i \in \Lambda$ choose $y \in \partial B\left(x_{2}, 7 r / 2\right)$ with $u_{i}(y)=-M\left(7 r / 2,-u_{i}, x_{2}\right)$ and put

$$
w(x)=u_{i}(x)+M\left(\frac{7}{2} r,-u_{i}, x_{2}\right)
$$

when $x \in B\left(y, \frac{1}{2} r\right)$. As in (2.9), we deduce from (2.2)-(2.3) of Lemma 3 that

$$
M(t, w, y) \leq c\left(\frac{t}{r}\right)^{\alpha} M\left(\frac{1}{2} r, w, y\right), \quad 0<t<\frac{1}{4} r
$$

Using (3.8) we obtain

$$
M\left(\frac{1}{2} r, w, y\right) \leq c M\left(\frac{7}{2} r,-u_{i}, x_{2}\right)
$$

and thereupon from (3.9) that there exists $c_{0}, 0<c_{0} \leq \frac{1}{4}$, such that

$$
M\left(c_{0} r, w, y\right) \leq \frac{1}{2} M\left(\frac{7}{2} r,-u_{i}, x_{2}\right) .
$$


Equivalently,

$$
u_{i}(x) \leq-\frac{1}{2} M\left(\frac{7}{2} r,-u_{i}, x_{2}\right) \leq-\frac{1}{2} M\left(3 r,-u_{i}, x_{2}\right)<-\lambda,
$$

when $x \in B\left(y, c_{0} r\right)$, thanks to (3.8). Hence, there exists $c_{8}=c_{8}\left(n, a, b, p_{1}, p_{2}\right)>0$ such that

$$
c_{8}\left|\left\{x \in B\left(x_{2}, 4 r\right):-u_{i}(x)>\frac{1}{2} M\left(3 r,-u_{i}, x_{2}\right)>\lambda\right\}\right| \geq r^{n},
$$

which is (1.15) for $i \in \Lambda$. From (i) we conclude first that $m \leq c_{3}$ and thereupon that Theorem 1 is true.

To prove Corollary 1 fix $i, 1 \leq i \leq m$ and observe as in (2.9) and (3.9) that it follows from (2.2)-(2.3) of Lemma 3 for $0<r<\rho$,

$$
\underset{B(0, r)}{\operatorname{osc}} u_{i} \leq c\left(\frac{r}{\rho}\right)^{\alpha} \underset{B(0, \rho)}{\operatorname{osc}} u_{i} \leq c\left(\frac{r}{\rho}\right)^{\alpha} M\left(2 \rho, u_{i}-u_{i}(0), 0\right) .
$$

Letting $\rho \rightarrow \infty$, we conclude that either

$$
\lim _{\rho \rightarrow \infty} \rho^{-\alpha} M\left(\rho, u_{i}, 0\right)=+\infty,
$$

or $u_{i} \equiv u_{i}(0)$. Since $u_{i}$ is nonconstant, it follows that $(3.10)$ holds. Hence, $M\left(R / 100, u_{i}, 0\right)>c_{3} \lambda$ for sufficiently large $R$, and so $m \leq c_{3}$, thanks to Theorem 1 .

Finally, we point out that some examples of Rickman imply the existence of $u_{1}, \ldots, u_{m}$, satisfying the hypotheses of Theorem 1 with $\lambda=0$ and $m>1$. Indeed, Rickman in [16, Theorem 1.2] constructed an example of a $K$ quasiregular mapping $f$ which omits $a_{1}, \ldots, a_{m}(m>2)$. In fact, $m=m(K) \rightarrow \infty$ as $K \rightarrow \infty$. Let

$$
u=\log \left|f(x)-a_{1}\right|-\log \left|f(0)-a_{1}\right|,
$$

and note from Lemmas $1-2$, as in (1.12)-(1.13), that there exists a sequence $\left\{B\left(y_{k}, 2 r_{k}\right)\right\}_{1}^{\infty}$ with $u\left(y_{k}\right)=0$,

$$
M\left(5 r_{k}, u, y_{k}\right) \leq c M\left(2 r_{k}, u, y_{k}\right),
$$

and $M\left(2 r_{k}, u, y_{k}\right) \rightarrow \infty$, as $k \rightarrow \infty$. Put

$$
u_{i}^{(k)}(x)=\frac{\log \left|f\left(y_{k}+4 r_{k} x\right)-a_{i}\right|}{M\left(4 r_{k}, u, y_{k}\right)} .
$$

when $x \in B(0,1), 1 \leq i \leq m$, and $k=1,2, \ldots$ From (2.3) of Lemma 3 we see that $\left|u_{i}^{(k)}\right| \leq c$ in $\bar{B}(0,1)$ for $1 \leq i \leq m$, with at most one possible exception. Also $u_{i}^{(k)} \in F(a, b, n, n, B(0,1))$ for some $a, b$ independent of $i, k$, as follows from the fact that $f$ is quasiregular. Using (2.2) and Ascoli's theorem, we find that a subsequence of $\left(u_{i}^{(k)}\right)$ converges uniformly on $\bar{B}(0,1)$ to $u_{i} \in F(a, b, n, n, B(0,1))$, whenever $i \in \Lambda$, where $\Lambda$ has cardinality at least $m-1$. Clearly $u_{i}, i \in \Lambda$ satisfies (i)-(iii) of Theorem 1 with $\lambda=0$. Moreover, $u_{i} \not \equiv 0, i \in \Lambda$, thanks to (3.11). 


\section{References}

[1] Bojarski, B., and T. Iwaniec: Analytical foundations of the theory of quasiconformal mappings in $R^{n}$. - Ann. Acad. Sci. Fenn. A I Math. 8, 1983, 257-324.

[2] Eremenko, A., and M. Sodin: Second main theorem of value distribution theory for meromorphic curves and non-linear divisors. - Dokl. Acad. Nauk USSR number, 1990, 1293-1295 (Russian).

[3] Eremenko, A., and M. Sodin: Value distribution of meromorphic functions and meromorphic curves from the point of view of potential theory. - Leningrad J. of Math. (to appear).

[4] ERemenko, A.: A new proof of Drasin's theorem on meromorphic functions of finite order with maximal deficiency sum, 1,2. - Function Theory, Functional Analysis and Their Applications 44, 1985, 48-52 (Russian).

[5] Garofalo, N., and F. Lin: Monotinicity properties of variational integrals, $A_{p}$ weights, and unique continuation. - Indiana U. Math. J, 35, 1986, 245-268.

[6] Heinonen, J., and T. KilpeläInEN: Superharmonic functions and supersolutions of degenerate elliptic equations. - Ark. Mat.26, 1988, 87-105.

[7] Kenig, C.: Restriction theorems, Carleman estimates, uniform Sobolev inequalities, and unique continuation. - Harmonic analysis and partial differential equations, Lecture Notes in Mathematics 1384. Springer-Verlag, 1987, 69-90.

[8] Kinderlehrer, D., and G. Stampacchia: An introduction to variational inequalities and their applications. - Academic Press, 1980.

[9] KilpeläInen, T., and J. Maly: Degenerate elliptic equations with measure data and nonlinear potentials. - To appear.

[10] Martio, O.: Counterexamples for unique continuation. - Manuscripta Math. 60, 1988, 21-47.

[11] Michael, J., and W. Ziemer: Interior regularity for solutions to obstacle problems. Nonlinear Anal. 10, 1986, 1427-1448.

[12] Rickman, S.: On the number of omitted values of entire quasregular mappings. - J. Analyse Math. 37, 1980, 100-117.

[13] Rickman, S.: A defect relation for quasimeromorphic mappings. - Ann. of Math. 114, 1981, 165-191.

[14] Rickman, S.: Value distribution of quasiregular mappings. - Lecture Notes in Mathematics 981. Springer-Verlag, 1981, 220-245.

[15] Rickman, S.: Quasiregular mappings and metrics on the $n$ sphere with punctures. Comment. Math. Helv. 59, 1984, 136-147.

[16] Rickman, S.: The analogue of Picard's theorem for quasiregular mappings in dimension three. - Acta Math. 154, 1985, 195-242.

[17] Serrin, J.: Local behavior of solutions of quasi-linear equations. - Acta Math. 111, 1964, $247-302$.

[18] WolfF, T.: Unique continuation for $|\Delta u| \leq V|\nabla u|$ and related problems. - To appear.

\author{
Alexandre Eremenko \\ Purdue University \\ Department of Mathematics \\ West Lafayette, Indiana 47907 \\ U.S.A.
}

Received 18 January 1991
John L. Lewis

University of Kentucky

Department of Mathematics

Lexington, Kentucky 40506-0027

U.S.A. 